\title{
SMS-Watchdog: Profiling Social Behaviors of SMS Users for Anomaly Detection
}

\author{
Guanhua Yan, Stephan Eidenbenz, and Emanuele Galli \\ Information Sciences (CCS-3) ${ }^{\star}$ \\ Los Alamos National Laboratory \\ \{ghyan, eidenben, egalli\}@lanl.gov
}

\begin{abstract}
With more than one trillion mobile messages delivered worldwide every year, SMS has been a lucrative playground for various attacks and frauds such as spamming, phishing and spoofing. These SMS-based attacks pose serious security threats to both mobile users and cellular network operators, such as information stealing, overcharging, battery exhaustion, and network congestion. Against the backdrop that approaches to protecting SMS security are lagging behind, we propose a lightweight scheme called $S M S$-Watchdog that can detect anomalous SMS behaviors with high accuracy. Our key contributions are summarized as follows: (1) After analyzing an SMS trace collected within a five-month period, we conclude that for the majority of SMS users, there are window-based regularities regarding whom she sends messages to and how frequently she sends messages to each recipient. (2) With these regularities, we accordingly propose four detection schemes that build normal social behavior profiles for each SMS user and then use them to detect SMS anomalies in an online and streaming fashion. Each of these schemes stores only a few states (typically, at most 12 states) in memory for each SMS user, thereby imposing very low overhead for online anomaly detection. (3) We evaluate these four schemes and also two hybrid approaches with realistic SMS traces. The results show that the hybrid approaches can detect more than $92 \%$ of SMSbased attacks with false alarm rate $8.5 \%$, or about two thirds of the attacks without any false alarm, depending on their parameter settings.
\end{abstract}

Keywords: SMS, anomaly detection, relative entropy, JS-divergence.

\section{Introduction}

The Short Message Service (SMS) provided by cellular carriers is a connectionless message transfer service with low capacity. Since its inception in December 1992, when the first short message was delivered in the Vodafone GSM network in the United Kingdom [12, SMS has been growing at a blistering speed. According to the IDC research firm, the total number of SMS subscribers in the US in 2006 was estimated at 102 million and is expected to reach 184 million in 2011; the number of short messages delivered in the US will grow at an even faster pace,

\footnotetext{
^ Los Alamos National Laboratory Publication No. LA-UR 08-07637.
} 
which will jump from 157 billion in 2006 to 512 billion in 2011 [22]. Another report by Gartner shows that the Asia-Pacific region, whose SMS subscribers sent 1.5 trillion short messages in 2007, is leading the SMS growth over North America and Western Europe, whose SMS subscribers generated 189 and 202 billion short messages in 2007, respectively [18.

Due to its increasing popularity, SMS has become a lucrative target for fraudulent behaviors that have been rampant in the Internet for decades. For instance, 88 percent of mobile users in China 4] have been plagued by SMS spams. Meanwhile, the convergence of the telecommunication world and the Internet has led to the emergence of SMS phishing, also dubbed "SMiShing", which could steal confidential account information from mobile devices [16] or spreading mobile malware 15. SMS spoofing is another type of attacks based on SMS: by manipulating address information in SMS messages, an SMS spoofing attack simulates the behavior of a legitimate mobile device so that foreign networks (as opposed to the home network of the mobile device) mistakingly think these messages originate from that device. SMS spoofing attacks have been launched against major cellular operators in Europe in the past [19. Besides these spamming, phishing and spoofing attacks, other SMS-based attacks include SMS flooding, which aims to overload the cellular network, and SMS faking, which mimics the behavior of an SMS switch to send messages [17. In particular, SMS flooding could shut down cellular services entirely in a large area [6].

SMS-based attacks pose serious security threats to both mobile users and cellular networks, including information stealing, overcharging, battery exhaustion, and network congestion. Effective countermeasures, unfortunately, are still lagging behind. Many existing solutions are aimed at detecting malware on mobile devices with techniques inspired by their counterparts in IP networks. For instance, signature-based detection schemes are proposed to examine mobile network traffic [8] or power usage of mobile applications [9] for signatures that are extracted from existing mobile malware instances. A machine learning-based approach is developed in [1] to catch mobile malware by discriminating behaviors of normal applications and malware at the level of system events and API calls.

Although effective against some SMS-based attacks, these mobile malware detection approaches have their flip sides. First, not all aforementioned SMS-based attacks originate from mobile malware. For instance, SMS spoofing usually comes from a device that simulates an authentic mobile handheld to fool a foreign network. Second, many of these approaches demand extra computational resources on mobile devices, which accelerate exhaustion of their batteries. Third, operating systems of existing mobile devices often lack sophisticated countermeasures to prevent mobile malware from disabling device-resident detection schemes.

In this work, we take a different avenue to detect anomalous SMS behaviors. We propose a detection framework called $S M S$-Watchdog, which is deployed at a place where a mobile user's short message records can be easily accessed, such as the Short Messaging Service Center (SMSC) in a typical SMS architecture. Hence, our work alleviates typical shortcomings of device-resident mobile detection schemes, such as extra power consumption for detection and inability to 
catch spoofed short messages. Motivated by observations made from a real-world SMS dataset, our work exploits regularities inherent in a typical user's SMS behaviors for anomaly detection. Our key contributions in this paper are summarized as follows: (1) After analyzing an SMS trace collected within a five-month period, we conclude that for the majority of SMS users, there are window-based regularities regarding whom she sends messages to and how frequently she sends messages to each recipient. (2) With these regularities, we accordingly propose four detection schemes that build normal social behavior profiles for each SMS user and then use them to detect SMS anomalies in an online and streaming fashion. Each of these schemes stores only a few states (typically, at most 12 states) in memory for each SMS user, thereby imposing very low overhead for online anomaly detection. (3) We evaluate these four schemes and also two hybrid approaches with realistic SMS traces and the results show that the hybrid approaches can detect more than $92 \%$ of SMS-based attacks with false alarm rate $8.5 \%$, or two thirds of the attacks without any false alarm, depending on their parameter settings.

Related work. In [27, Zerfos et al. used an SMS trace collected from a national cellular carrier in India to examine message size distribution, message service time distribution, and thread-level characteristics. The same trace was later investigated by Meng et al. to understand delivery reliability and latency [13. It is noted that their trace, although containing more SMS users than the one analyzed in this paper, lasts only three weeks. By contrast, our trace contains short messages within five months, thus offering insights on long-term and persistent behaviors of SMS users. Enck et al. showed that SMS flooding from the Internet side can cause severe denial-of-service attacks against cellular network operations in a large area [6] and later they proposed some countermeasures to prevent such attacks [24]. Our work in this paper attempts to identify anomalous SMS behaviors by comparing them against normal SMS user's social behavior profiles and is thus complementary to their work.

Techniques based on profiling human social behaviors have been applied to detect anomalies in other types of network traffic. For instance, Stolfo et al. developed a data mining system that builds behavioral profiles or models with methods such as user cliques, Hellinger distance, and cumulative distributions for emails users [20]. Using injected simulated viral emails, they show that the detection system performs well with high accuracy. In [26], Yan et al. applied change-point detection techniques to detect worm propagation in IM (Instant Messaging) networks. They show that schemes simply counting the number of instant messages sent by each user can easily be circumvented by a carefully crafted IM worm. Observing that the distribution of the number of messages sent to each contact on the buddy list is typically highly skewed, they developed an effective technique that periodically calculates the overall likelihood that instant messages are sent to each recipient and look for abrupt changes to detect IM worm propagation. Moreover, in this work we use information-theoretical measures to detect anomalies in SMS traffic and these techniques have been applied to detect anomalies in Internet traffic before 11 14. Besides focusing on a 
different type of network traffic, we also address the scalability issues that were not considered in previous work. For a more comprehensive survey on anomaly detection techniques and their applications, we refer interested readers to [2].

Anomaly detection for mobile phone networks has a long history. For instance, calling activities have been examined to detect mobile phone fraud [7] 23] [5] and mobility patterns of mobile devices have been profiled to detect cloning attacks and cell phone losses 25] 21. Recently, due to the increasing popularity of smart phones, a growing number of malware instances have been observed on these mobile devices. Many existing approaches to detect mobile malware work by profiling behaviors of normal applications [9] [1] our work instead focuses on profiling normal SMS user behaviors.

Organization. The remainder of the paper is organized as follows. Section 2 briefly introduces typical SMS architectures and how we collect the SMS trace. In Section 3, we first analyze SMS user behaviors in the trace and discuss what statistic metrics exhibit low variation. We then discuss the design of SMSWatchdog in Section 4. Based on the results from the trace analysis in Section [3. we propose four different detection schemes in Section 5. We evaluate the performance of these four schemes and also two hybrid schemes in Section 6. We finally make concluding remarks and discuss the scope of our work in Section 7

\section{Background on SMS and SMS Traces}

SMS architecture. SMS is a service that provides a connectionless transfer of messages with at most 160 characters using signaling channels in cellular networks. Figure 1 illustrates the basic SMS architecture in a GSM-based system. A short message sender (on the right bottom corner) uses an originating $M S$ (Mobile Station) to send a short message to a receiver (on the left bottom corner in Figure 1). The short message is delivered to a nearby BSS (Basestation System) through the GSM signaling channel and then the MSC (Mobile Switching Center) associated with this BSS. The MSC first checks with a VLR (Visitor Location Register) database whether the originating MS is allowed to receive

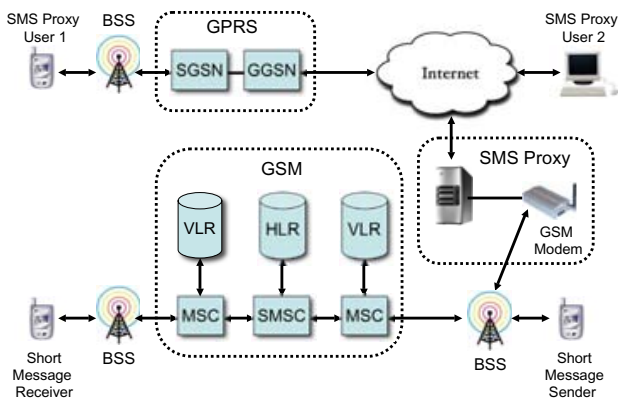

Fig. 1. SMS Architecture and SMS Proxy 
the short message service. The VLR database temporarily stores subscription information for the visiting mobile stations so that the associated MSC knows what services should be provided to them. If the originating MS is allowed to use SMS, the MSC further routes the short message to SMSC, a dedicated storeand-forward server that handles SMS traffic.

The SMSC is responsible for forwarding the short message to the targeted mobile device, also called terminating $M S$ (on the left bottom corner). To do that, it queries an HLR (Home Location Register) database, which keeps information about cellular subscribers, such as their profile information, current location, billing data, and validation period. The HLR responds by sending back the serving MSC address of the terminating MS. Thereafter, the SMSC forwards the short message to that MSC, which further queries its associated VLR database for the location area of the terminating MS. Once the location of the terminating MS is found, the short message is delivered to it through its nearby BSS.

SMS trace collection. The SMS trace used in this work was collected from an SMS proxy in Italy. This SMS proxy connects with the Internet, and also the GSM network through a GSM modem. There are two classes of users for this SMS proxy. Similar to the regular SMS sender, the first class (e.g., User 1) also use an MS to send short messages. These short messages, however, do not use GSM signaling channels; instead, they are delivered through the GPRS network to the SMS proxy, which further forwards these messages to their recipients through the GSM modem. The second class of SMS proxy customers (e.g., user 2) send their short messages to the SMS proxy through the Internet and then to their receiving MSes through the GSM modem. The economic incentive for such an SMS proxy is the price difference between regular SMS and GPRS messages.

The SMS proxy was launched in early 2008 and we obtained communication logs of all its users from April 15, 2008 to September 14, 2008. Through this period, there were 2,121 users that have used it to send short messages. In total, 146,334 short messages have been sent through this proxy. As this data trace covers a large number of users, we believe that it is representative of general SMS traffic. Due to its short history, the SMS proxy has not been seen suffering malicious attacks yet. Hence, analysis on the data collected sheds light on how normal SMS users behave socially. Moreover, the long time span of this trace enables us to investigate the persistent behavioral patterns of SMS users. This is contrast to previous work which mainly focus on analyzing communication traces of SMS users only within a short period of time [27] 13].

\section{Trace Analysis}

In this section, we analyze the dynamics of the system from which the trace was collected and then derive regularities inherent in behaviors of normal SMS users.

System dynamics. In Figure 2, we show the number of short messages that have been observed by the SMS proxy each day. From the graph, we observe an 


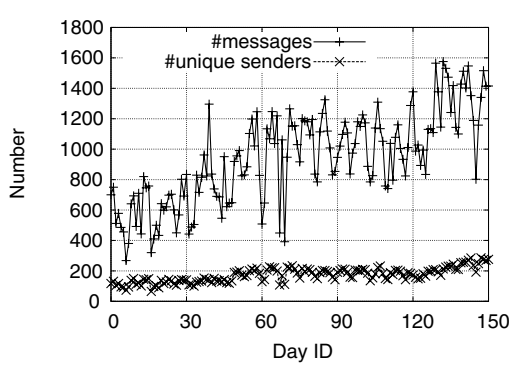

Fig. 2. Number of messages and number of unique senders per day

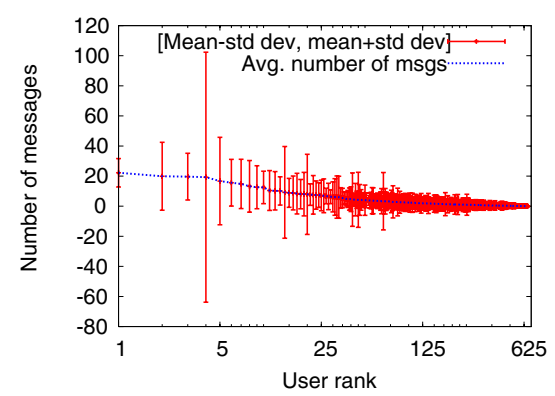

(1) Daily

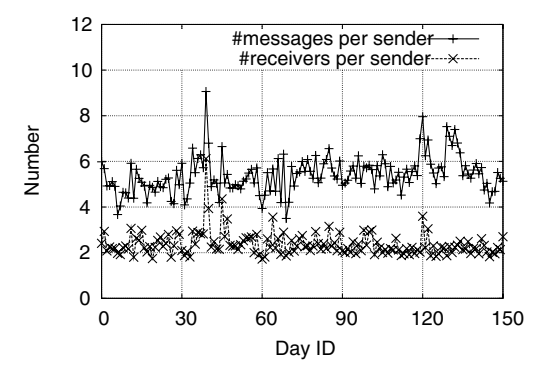

Fig. 3. Number of messages and receivers per sender per day

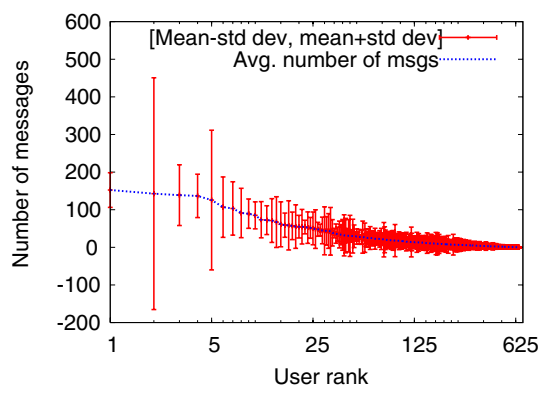

(2) Weekly

Fig. 4. Average numbers of messages for persistent users

obvious trend that an increasing number of short messages have been transmitted through the SMS proxy. For instance, the number of short messages observed has increased by $67 \%$ from May to August. This is attributed to the growing number of users of the system during the trace collection period. Figure 2 also depicts the number of users that sent at least one message in a day. In August, there were 972 active customers, as opposed to only 662 ones in May.

Although the number of users in the system was not stationary during the data collection period, we find that both the number of messages sent out by each user and the number of receivers per sender each day are quite stationary. They are illustrated in Figure 3, from which we observe that each day an active user sends about 5.4 messages to 2.4 recipients on average. We also note that some users in the dataset used the SMS proxy to send short messages for only a short period of time. As we are only interested in persistent behaviors of SMS users, we do not consider these temporary users. From the dataset, we obtain a list of 662 users whose first and last short messages were sent at least 60 days apart. For brevity, we call them persistent users, who contributed about $75 \%$ of the entire set of short messages.

Temporally periodic behaviors of persistent users. We are interested in statistically time-invariant metrics that characterize behaviors of SMS users so 


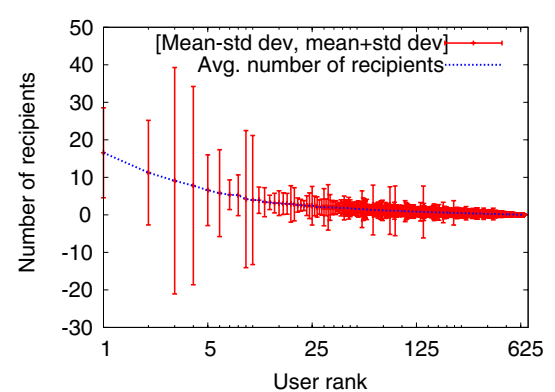

(1) Daily

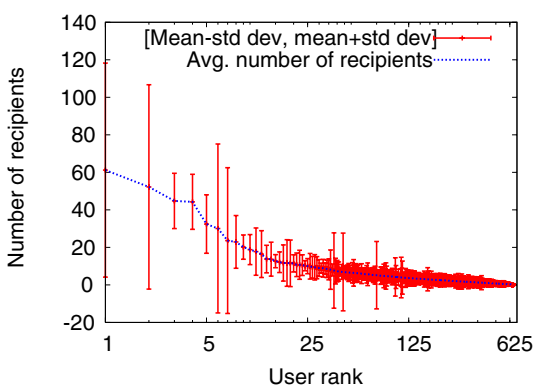

(2) Weekly

Fig. 5. Average numbers of recipients for persistent users

that they can be applied for anomaly detection. An ideal metric should have low variation, which helps reduce false alarm rates. As human behaviors such as lunch and shopping often exhibit temporal periodicity, a natural hypothesis is that SMS user behaviors should follow similar patterns. To verify this, we depict in Figure 4 the average number of messages sent out per day and per week by each persistent user versus his overall rank. Note that the $\mathrm{x}$-axis, which indicates the user rank, is shown in logarithmic scale. For each persistent user in the graphs, we also show the range between the mean plus and minus one standard deviation. Clearly, these graphs reveal that both daily and weekly numbers of messages sent by persistent users exhibit high variation for many users.

A better way of quantifying the variation of a statistic metric is Coefficient of Variation (COV), defined as the ratio of the standard deviation to the mean. Generally speaking, a distribution with $C O V<1$ portends low variation, and those with $C O V>1$ are considered high variation. Among all the persistent users, $97.7 \%$ and $71.9 \%$ of them have COVs $>1$ for the daily and weekly number of short messages they sent, respectively, suggesting that neither of these two metrics is good for anomaly detection.

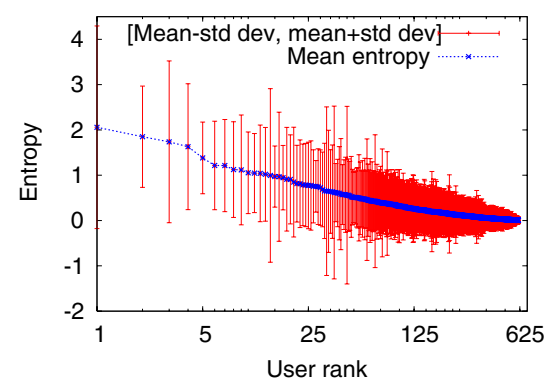

(1) Daily

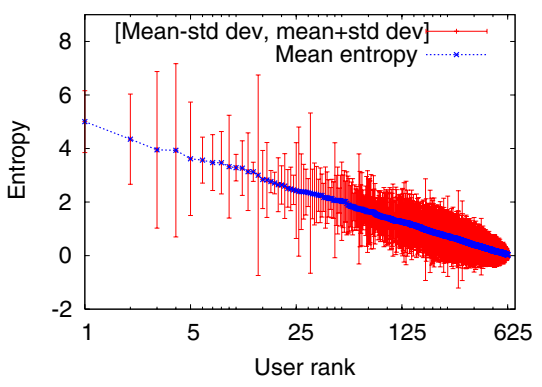

(2) Weekly

Fig. 6. Average entropies for persistent users 
We perform a similar analysis on the daily and weekly number of unique recipients to whom persistent users sent their short messages, and the results are provided in Figure 5. We observe that $94.4 \%$ and $54.4 \%$ of persistent users have COVs $>1$ for their daily and weekly number of unique recipients, respectively. Hence, they are not good candidates for anomaly detection either.

We further analyze the entropy of the distribution of the number of short messages sent to each unique recipient for every persistent SMS user. The entropy, $H$, is defined as: $H=-\sum_{i=1}^{n} p_{i} \times \log _{2} p_{i}$, where $p_{i}$ is the fraction of short messages sent to the $i$-th unique recipient in a day or a week (suppose that there are $n$ unique recipients). Figure 6 shows the average daily and weekly entropies for each persistent SMS user. Similar to the other metrics that we have studied, these two also show high variation: $98.0 \%$ and $66.9 \%$ of persistent users have COVs $>1$ for their daily and weekly entropies, respectively. Therefore, neither metric seems plausible for anomaly detection.

Window-based behaviors of SMS users. The above analysis reveals that high variation is inherent in many SMS users' behaviors on a temporally periodic basis. We now examine their behaviors from a window-based perspective. For each SMS user in the dataset, we form $m$ blocks, each of which contains an equal number of successive short messages. Given the sequence of blocks from the same SMS sender, we first consider the number of unique recipients to whom messages in each block are sent. Similar to our previous analysis, we are interested in the variation of this metric. To ensure that there are enough short messages in each block, we consider only users that have sent at least $\theta$ short messages. In our study, we consider two $\theta$ values, 100 and 200, which lead to a set of 353 and 167 qualified SMS users, respectively.

Figure 7 gives the average number of unique recipients when $\theta$ is 200 (we have similar results for $\theta=100$, but due to space limitation, we do not show them here). Different from the metrics characterizing temporally periodic behaviors, the number of unique recipients seen in each window seems to have low variation. This is confirmed by Table 1, which shows that the COV exceeds 1.0 for less than $1 \%$ of the users, regardless of which $\theta$ and $m$ are used.

For each SMS user, we also consider the entropy of the distribution of the number of messages sent to each unique recipient within each block. Figure 8 depicts the mean for $\theta=200$, and Table 1 also provides the average COV in different combinations of $\theta$ and $m$. In all cases, the average COV is smaller than $20 \%$. It also seems that the COV can be reduced by either increasing the threshold $\theta$ or choosing a smaller $m$.

These results reveal that window-based behaviors of SMS users bear lower variation than their temporally periodic behaviors. In the following discussion, we further explore the similarity across different blocks for each SMS user.

Similarity measures. First, we study the similarity in the set of recipients between different blocks for the same SMS user. For the $i$-th block $\mathcal{B}_{i}$ associated with an SMS user, we let $\mathcal{R}_{i}$ denote the entire set of unique recipients of the short messages in this block. We use the following recipient similarity metric to 
Table 1. Fraction of users with $\mathrm{COV}>1$ regarding window-based behaviors

\begin{tabular}{c|cc|cc}
\hline \multirow{2}{*}{$\mathrm{m}$} & \multicolumn{2}{|c|}{$\theta=100$} & \multicolumn{2}{c}{$\theta=200$} \\
\cline { 2 - 5 } & \#Recipients & Entropy & \#Recipients & Entropy \\
\hline \hline 10 & $0.3 \%$ & $14.5 \%$ & $0.6 \%$ & $11.2 \%$ \\
20 & $0.3 \%$ & $18.1 \%$ & $0.6 \%$ & $15.6 \%$ \\
\hline
\end{tabular}

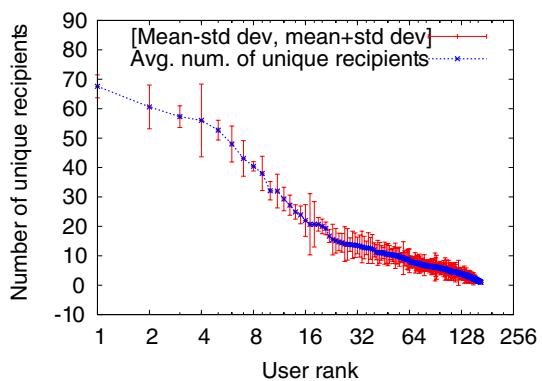

(1) $m=10, \theta=200$

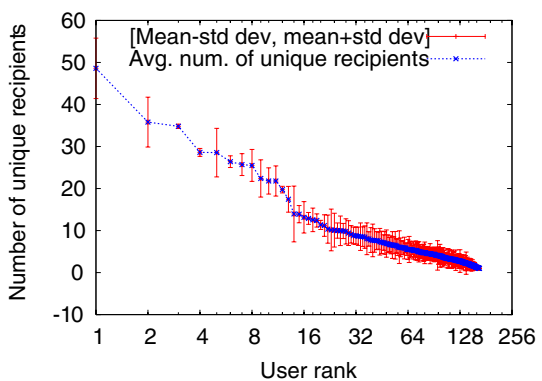

(2) $m=20, \theta=200$

Fig. 7. Average number of unique recipients

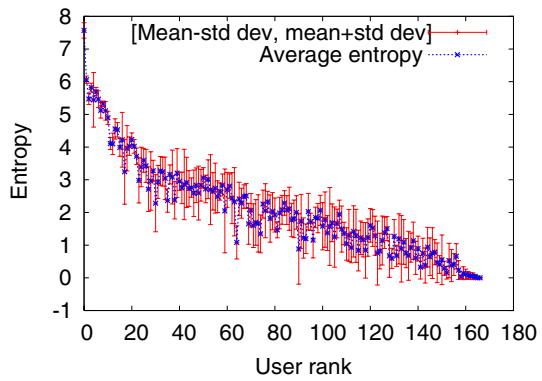

(1) $m=10, \theta=200$

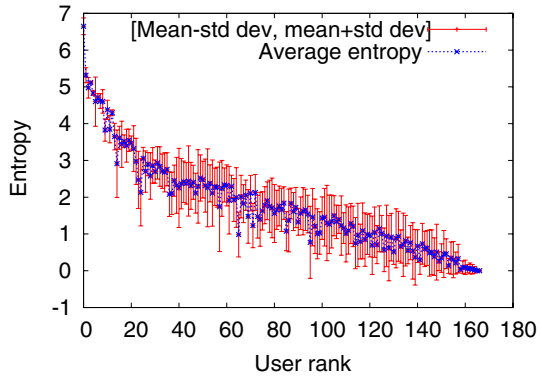

(2) $m=20, \theta=200$

Fig. 8. Average entropies

measure the distance between two sets $\mathcal{R}_{i}$ and $\mathcal{R}_{j}(i \neq j)$ :

$$
S\left(\mathcal{R}_{i}, \mathcal{R}_{j}\right)=\frac{\left|\mathcal{R}_{i} \cap \mathcal{R}_{j}\right|}{2} \times\left(\frac{1}{\left|\mathcal{R}_{i}\right|}+\frac{1}{\left|\mathcal{R}_{j}\right|}\right) .
$$

$S\left(\mathcal{R}_{i}, \mathcal{R}_{j}\right)$ gives the average fraction of common elements that are shared between sets $\mathcal{R}_{i}$ and $\mathcal{R}_{j}$. Clearly, $S\left(\mathcal{R}_{i}, \mathcal{R}_{j}\right)$ close to 0 means that $\mathcal{R}_{i}$ and $\mathcal{R}_{j}$ share few common elements and vice versa if it is close to 1 . For each SMS user, we call set $\left\{S\left(\mathcal{R}_{i}, \mathcal{R}_{j}\right): \forall i, j, i \neq j\right\}$ as her recipient similarity metric set.

Figure 9 depicts the recipient similarity metrics with $\theta=200$. One observation is that SMS users differ significantly on how they send messages regularly: for some users they send short messages to almost the same set of recipients, but for 


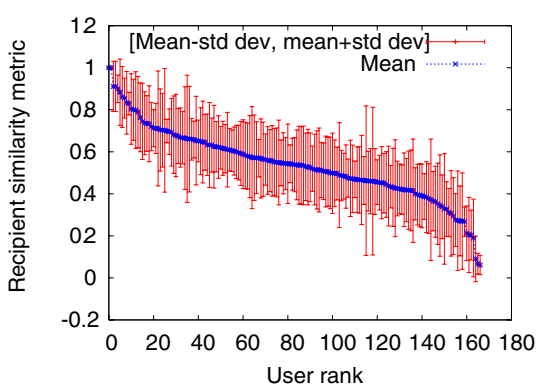

(1) $m=10$

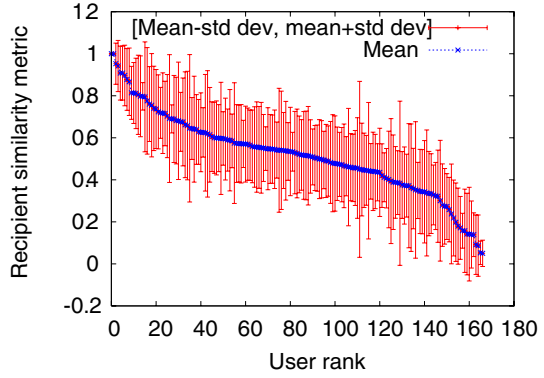

(2) $m=20$

Fig. 9. Recipient similarity metric for all recipients $(\theta=200)$

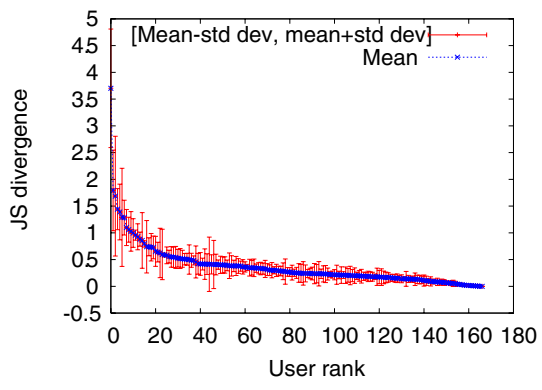

(1) $m=10$

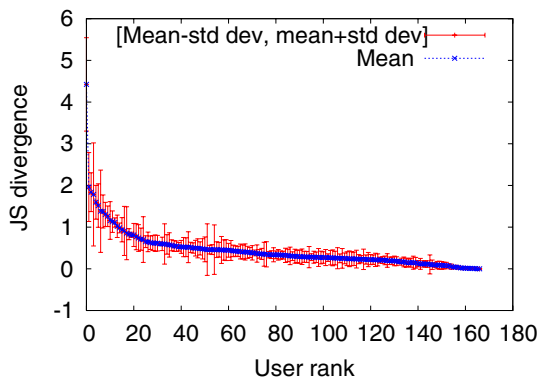

(2) $m=20$

Fig. 10. JS-divergence for all recipients $(\theta=200)$

Table 2. Fraction of users with COV $>1$ regarding similarity measures for all recipients

\begin{tabular}{c|cc|cc}
\hline \multirow{2}{*}{$\mathrm{m}$} & \multicolumn{2}{|c|}{$\theta=100$} & \multicolumn{2}{c}{$\theta=200$} \\
\cline { 2 - 5 } & $\begin{array}{l}\text { Recipient } \\
\text { similarity divergence }\end{array}$ & $\begin{array}{l}\text { Recipient } \\
\text { similarity }\end{array}$ & $\begin{array}{c}\text { JS } \\
\text { sivergence }\end{array}$ \\
\hline \hline 10 & $4.5 \%$ & $4.3 \%$ & $0.6 \%$ & $1.2 \%$ \\
20 & $12.8 \%$ & $5.1 \%$ & $6.0 \%$ & $1.2 \%$ \\
\hline
\end{tabular}

some other: 1 they send short messages to a very diverse set of recipients. Given this fact, we thus cannot conclude that SMS users always tend to send short messages to the same set of users over time, which, if true, would be useful for anomaly detection. We further analyze the variation of the recipient similarity metric set for each SMS user and the results are given in Table 2. Interestingly, this measure exhibits low variation for the majority of the SMS users, implying that the recipients to whom most SMS users send short messages vary in a very similar fashion over time.

$\overline{1}$ These users typically send bulk messages for advertisement purposes. 
Previously we have used the entropy to measure the uncertainty of the distribution of the number of short messages sent to each recipient. A natural question extended from that is how similar these distributions are across different messages blocks. A metric commonly used for this is relative entropy, also called Kullback-Leibler $(K L)$ divergence [3], which is defined as follows:

$$
D_{\mathrm{KL}}(P \| Q)=\sum_{i} P(i) \log \frac{P(i)}{Q(i)},
$$

where $P$ and $Q$ are two distributional functions.

Note that $D_{K L}(P \| Q)$ is undefined if the support 2 of $P$ is not a subset of the support of $Q$. Hence, directly applying the relative entropy here is problematic because an SMS user may have different sets of recipients in two message blocks. Moreover, relative entropy is not symmetric, i.e., $D_{K L}(P \| Q)$ may not equal $D_{K L}(Q \| P)$. Due to these constraints, we instead use the Jensen-Shannon (JS) divergence [10], whose computation relies on the KL-divergence:

$$
D_{\mathrm{JS}}(P \| Q)=\frac{1}{2}\left[D_{\mathrm{KL}}\left(P \| \tilde{A}_{P, Q}\right)+D_{\mathrm{KL}}\left(Q \| \tilde{A}_{P, Q}\right)\right],
$$

where function $\tilde{A}_{P, Q}$ denotes the average distribution: $\tilde{A}_{P, Q}(x)=(P(x)+Q(x)) / 2$. Obviously, $D_{\mathrm{JS}}(P \| Q)$ is always defined and also symmetric.

Figure 10] shows the JS-divergences when $\theta=200$. We observe that the JSdivergence also varies significantly among different SMS users. It is clear that the JS-divergence has low variation for the majority of the SMS users, which is confirmed by Table 2. for all four combinations of $\theta$ and $m$, less than $6 \%$ percent of the SMS users have a JS-divergence COV greater than 1.

Next, we show how the top five recipients receiving the most messages correlate with each other between different blocks. Figure 11 depicts the recipient similarity metrics for the top 5 recipients with $\theta=200$. Still, this metric varies significantly among different SMS users. The fractions of SMS users with $C O V>1$ are shown in Table 3. Clearly, these fractions are higher than their counterparts where all recipients are considered, but are still relatively small.

Similarly, we study the JS-divergence of the distributions of the numbers of messages sent to the top five recipients among different blocks. Here, we normalize the probability that each of those top five recipients receives a message by dividing it by the probability that any of those top five recipients receives

\begin{tabular}{|c|c|c|}
\hline \multirow[t]{2}{*}{$\mathrm{m}$} & $\theta=100$ & $\theta=200$ \\
\hline & $\begin{array}{l}\text { Recipient } \quad \text { JS } \\
\text { similarity } \\
\end{array}$ & \begin{tabular}{|l|l} 
Recipient $\quad$ JS \\
similarity divergence
\end{tabular} \\
\hline 10 & $8.2 \%$ & $6.6 \%$ \\
\hline 20 & $13.6 \%$ & $12.6 \%$ \\
\hline
\end{tabular}

Table 3. Fraction of users with COV $>1$ for top-5 recipients (similarity measure)

$\overline{2}$ The support of a function is the set of points where the function is not zero. 


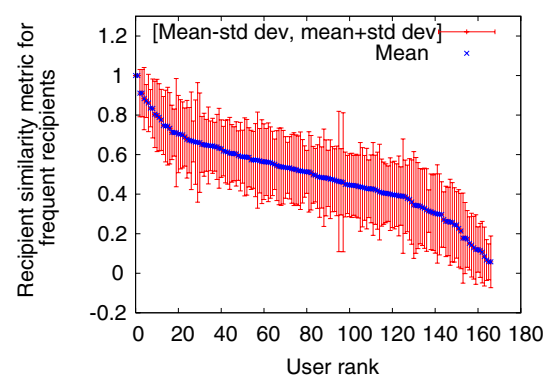

(1) $m=10$

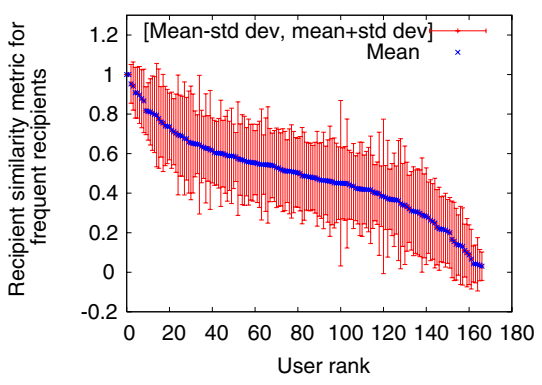

(2) $m=20$

Fig. 11. Recipient similarity metric for top 5 recipients $(\theta=200)$

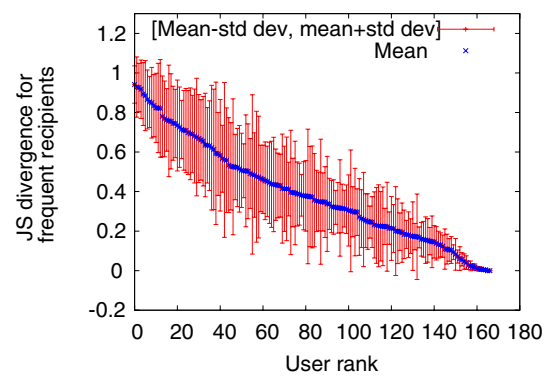

(1) $m=10$

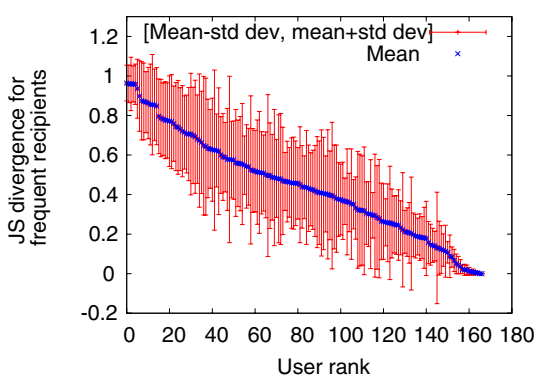

(2) $m=20$

Fig. 12. JS-divergence for top 5 recipients $(\theta=200)$

a short messages. For instance, if the top five recipients receive $5,4,3,2$, and 1 short messages within a window, the normalized probabilities are $1 / 3,4 / 15$, $1 / 5,2 / 15$, and $1 / 15$, respectively.

The JS-divergence of the distributions for the top five recipients is shown in Figure 12 for $\theta=200$. We notice that the average JS-divergence is always no higher than 1 and the fractions of SMS users with COV $>1$ exhibit a similar pattern as the recipient similarity metric: although they are higher than their counterparts where all recipients are considered, they are still very small.

In summary, different SMS users may have different levels of similarity across their message blocks, but the level of similarity across different message blocks of the same SMS user typically does not change significantly.

\section{SMS-Watchdog Design}

In the following, we shall discuss how to exploit the regularities inherent in social behaviors of SMS users for anomaly detection. Before presenting the detailed algorithms, we first discuss two families of SMS-related attacks that are considered in this work and then present the design of SMS-Watchdog. 
Threat model. Two families of SMS-related attacks are considered here. The first type is called blending attacks, which occur when an SMS user's account is used to send messages for a different person. In reality, this can happen in three circumstances. First, a user's cell phone is implanted with a Trojan horse such that the cell phone can be remotely controlled to send messages for a different user ${ }^{3}$. Second, in an SMS spoofing attack, a fraudster can manipulate address information in messages to spoof a legitimate SMS user's identity in a foreign network. Third, if an SMS proxy is used (e.g., the one in Figure 1), an attacker can hack an SMS user's account at the front end and use it to send messages. All these attacks are termed as blending attacks because illegitimate messages are intermingled with legitimate ones from the detector's perspective. The second type of attacks, termed as broadcast attacks, mirrors the behavior of mobile malware that send out phishing or spamming messages to recipients that appear in normal ones. In such attacks, the mobile device from which these messages are sent have already been infected by the mobile malware.

Workflow of SMS-Watchdog. In our design, the SMS-Watchdog is placed at the SMSC, as shown in the SMS architecture in Figure1, which handles all SMS traffic for a specific cellular network. The workflow of SMS-Watchdog involves three steps:

(1) Monitoring: SMS-Watchdog maintains a detection window of size $h$ for each SMS user that has subscribed for this service. For the current detection window, it also keeps a counter $k$ for the number of sent SMS messages observed, and the sequence of recipients of these SMS messages. When $k$ becomes equal to $h$, SMS-Watchdog performs anomaly detection for this user as shown in Step (2).

(2) Anomaly detection: Given the recipients of the last $h$ SMS messages, the SMS-Watchdog checks whether there exist anomalous behaviors. If so, it raises an alert and goes to the next step. The detailed algorithms for anomaly detection will be presented in the next section.

(3) Alert handling: SMS-Watchdog sends an alert to the SMS user through a different communication channel, such as emails. Together with the alert, SMSWatchdog also sends to the user a summary of the last $h$ messages, such as the number of SMS messages per recipient and the whole time frame of these messages. The information is used to help the user to identify false positives. The user can first check whether the communication record shown on her mobile device matches with the summary sent by SMS-Watchdog within the given time frame. If the two do not match, it means that the user's SMS account has been spoofed and she can notify her service provider. Otherwise, the user further checks the summary to identify suspicious SMS behaviors. A more cautious user can even request to check the full communication record, regarding the

\footnotetext{
${ }^{3}$ To evade detection, the malware can delete the message from the "sent" folder after it is sent out; also, the message can attach a different returning number so that the recipient will not reply the message to the compromised phone.

${ }^{4}$ This can be automatically done with a software for the user's convenience.
} 
transmission time of each SMS message. If suspicious SMS behaviors have been observed, it is likely that the mobile device has been infected by malware and the user can use some anti-virus software to disinfect her mobile device.

\section{Anomaly Detection}

In this section, we provide the details on how Blue-Watchdog performs anomaly detection. The anomaly detection problem is formulated as follows: given an SMS user's communication history $\mathcal{V}=\left\{v_{1}, v_{2}, \ldots, v_{n}\right\}$, where $v_{i}(1 \leq i \leq n)$ denotes the recipient of the $i$-th short message in $\mathcal{V}$, and a test sequence $\mathcal{T}=$ $\left\{t_{1}, t_{2}, \ldots, t_{h}\right\}$, where $t_{j}(1 \leq j \leq h)$ denotes the recipient of the $j$-th short message in $\mathcal{T}$, is $\mathcal{T}$ anomalous?

Decision on detection window size $h$. We first address how to choose $h$, the detection window size for a specific user. As revealed in Section 3, a typical SMS user's window-based behaviors bear low variation in the number of unique recipients, entropy, recipient set similarity metric, and also JS-divergence, suggesting that choosing $h$ based on any of these metrics would be a possible solution. Compared with the other three metrics, however, the JS-divergence contains the most information, because its calculation depends on not only the set of recipients, the distribution of the number of short messages sent to each recipient, but also the distances between these distributions.

A feasible choice for $h$ is minimizing the COV of the JS-divergence after grouping sequence $\mathcal{V}$ by every $h$ short messages, because this can maximize the level of similarity among different blocks. Let $\operatorname{cov}(X)$ denote the COV of set $X$. We choose $h^{*}$ as follows:

$$
h^{*}=\underset{h_{\min } \leq h \leq h_{\max }}{\operatorname{argmin}} \operatorname{cov}\left(\left\{D_{J S}\left(P_{i} \| P_{j}\right) \mid 1 \leq i<j \leq\left\lfloor\frac{n}{h}\right\rfloor\right\}\right)
$$

where $P_{s}\left(1 \leq s \leq\left\lfloor\frac{n}{h}\right\rfloor\right)$ is the distribution of the number of short messages sent to each unique recipient within block $\left[v_{(s-1) h+1}, v_{(s-1) h+2}, \ldots, v_{s h}\right]$.

It is important to bound $h$ from both sides. On one hand, we need to ensure that the training sequence $\mathcal{V}$ is split into enough blocks so that the COV does not approach 0 (note that if $h=|\mathcal{V}|$, the COV is always 0 ). Also, a large $h$ induces high detection delay. On the other hand, we choose a sufficiently large $h$ to avoid performing anomaly detection too frequently. Hence, we constrain the selection of $h$ between $h_{\min }$ and $h_{\max }$, both of which are configurable parameters.

Mean-based anomaly detection. As both the average number of unique recipients and the average entropy within each block show low variation for most SMS users, the mean-based anomaly detection scheme identifies anomalous SMS behavior by checking whether the means of these two metrics in the test sequence $\mathcal{T}$ deviate significantly from the means observed from the history trace $\mathcal{V}$. Let $\left\{\mathcal{B}_{1}, \mathcal{B}_{2}, \ldots, \mathcal{B}_{l}\right\}$, where $l=\left\lfloor\frac{n}{h^{*}}\right\rfloor$, be the set of blocks after dividing sequence $\mathcal{V}$ by every $h^{*}$ messages. We use $R(\mathcal{X})$ and $H(\mathcal{X})$ to represent the number of unique recipients and the entropy of $\mathcal{X}\left(\mathcal{X}\right.$ can be a block $\mathcal{B}_{i}$ or the test sequence $\left.\mathcal{T}\right)$. 
We let $\mathbb{E}\left(R_{\mathcal{B}}\right)$ and $\operatorname{var}\left(R_{\mathcal{B}}\right)$ denote the mean and variance of $\left\{R\left(\mathcal{B}_{i}\right): 1 \leq i \leq l\right\}$, and $\mathbb{E}\left(H_{\mathcal{B}}\right)$ and $\operatorname{var}\left(H_{\mathcal{V}}\right)$ denote the mean and variance of $\left\{H\left(\mathcal{B}_{i}\right): 1 \leq i \leq l\right\}$.

We perform mean-based anomaly detection as follows: if $\left|R(\mathcal{T})-\mathbb{E}\left(R_{\mathcal{B}}\right)\right|>\alpha_{R}$, we raise an $R$-type alert (R-type detection); if $\left|H(\mathcal{T})-\mathbb{E}\left(H_{\mathcal{B}}\right)\right|>\alpha_{H}$, we raise an $H$-type alert (H-type detection). The rationale behind it is simple: if the mean observed from the test sequence deviates from the mean observed from the training trace by a predefined threshold, we deem it as anomalous.

Then, an important question is how to choose thresholds $\alpha_{R}$ and $\alpha_{H}$. A too large threshold may miss many anomalous behaviors but a too low threshold may raise too many false alerts. We do this based on the Chebyshev's inequality:

$$
\mathbb{P}\left\{\left|R(\mathcal{T})-\mathbb{E}\left(R_{\mathcal{B}}\right)\right|>\alpha_{R}\right\} \leq \frac{\operatorname{var}\left(R_{\mathcal{B}}\right)}{\alpha_{R}^{2}} .
$$

Let $\beta_{R}$ be the upper bound on the expected false alarm rate for R-type alerts. In practice, $\beta_{R}$ is a configurable input parameter. By having $\alpha_{R}=\sqrt{\operatorname{var}\left(R_{\mathcal{B}}\right) / \beta_{R}}$, we ensure that the expected false alarm rate of R-type alerts does not exceed $\beta_{R}$. Similarly, choosing $\alpha_{H}=\sqrt{\operatorname{var}\left(H_{\mathcal{B}}\right) / \beta_{H}}$, where $\beta_{H}$ gives the upper bound on the expected false alarm rate for $\mathrm{H}$-type alerts, renders the expected false alarm rate of $\mathrm{H}$-type alerts no greater than $\beta_{H}$.

From an implementation point of view, the mean-based anomaly detection scheme imposes trivial computational overhead. For each SMS user, it only requires only four states for anomaly detection: $\mathbb{E}\left(R_{\mathcal{B}}\right), \mathbb{E}\left(H_{\mathcal{B}}\right), \alpha_{R}$, and $\alpha_{H}$. The parameters can be derived from the training trace in an offline fashion, but their values can be stored in the memory (instead of on disk), thereby relieving the online anomaly detection from intensive disk access operations.

Similarity-based anomaly detection. We now explore how to exploit similarity-based metrics for anomaly detection. A naive implementation can be the following: we compute a similarity metric (recipient similarity metric or JSdivergence) between the test sequence $\mathcal{T}$ and each block in the history trace and check whether its mean significantly deviates from the mean similarity metric only between the blocks in the history trace. Although straightforward, this scheme demands knowledge of the whole history trace when performing online anomaly detection, thereby rendering it hardly practical due to its prohibitive computational cost.

Due to such performance concern, we propose a light-weight anomaly detection scheme as follows. First, instead of comparing the test sequence $\mathcal{T}$ against each block in the history trace, we condense information in the history trace into a set of recipients and a distributional function. Furthermore, we do not consider the entire set of recipients that have been witnessed in the history trace, but instead focus on the top few recipients that have received the most messages from the SMS user. Such simplification is justified by the previous results showing that the similarity metrics bear low variation even if only the top few recipients are considered within each message block.

Suppose that we only consider the top $\phi$ recipients. Let $\mathcal{G}_{\phi}(X)$ denote the set of the top $\phi$ recipients that receive the most messages within sequence $X$, and $\mathcal{Q}_{\phi}(X)$ the normalized distribution of the number of short messages sent 
to the top $\phi$ recipients within sequence $X$. The similarity-based anomaly detection scheme checks how significantly $S\left(\mathcal{G}_{\phi}(\mathcal{T}), \mathcal{G}_{\phi}(\mathcal{V})\right)$ and $D_{\mathrm{JS}}\left(\mathcal{Q}_{\phi}(\mathcal{T}) \| \mathcal{Q}_{\phi}(\mathcal{V})\right)$ deviate from the means that have been observed from the history trace.

Recall that $\left\{\mathcal{B}_{1}, \mathcal{B}_{2}, \ldots, \mathcal{B}_{l}\right\}$ is the set of blocks after dividing sequence $\mathcal{V}$ by every $h^{*}$ messages. To compute the means, we first define $\mathcal{V} \backslash \mathcal{B}_{i}$, where $1 \leq i \leq l$, as the sequence after block $B_{i}$ is removed from $\mathcal{V}$. We then let $\mathbb{E}\left(S_{\phi}\right)$ and $\operatorname{var}\left(S_{\phi}\right)$ be the mean and variance of elements in set $\left\{S\left(\mathcal{G}_{\phi}\left(\mathcal{B}_{i}\right), \mathcal{G}_{\phi}\left(\mathcal{V} \backslash \mathcal{B}_{i}\right)\right): 1 \leq i \leq\right.$ $l\}$. Similarly, we use $\mathbb{E}\left(D_{\phi}\right)$ and $\operatorname{var}\left(D_{\phi}\right)$ to denote the mean and variance of elements in set $\left\{D_{\mathrm{JS}}\left(\mathcal{Q}_{\phi}\left(\mathcal{B}_{i}\right) \| \mathcal{Q}_{\phi}\left(\mathcal{V} \backslash \mathcal{B}_{i}\right)\right): 1 \leq i \leq l\right\}$. Given $\mathcal{V}$ and $h^{*}$, we can easily calculate $\mathbb{E}\left(S_{\phi}\right), \operatorname{var}\left(S_{\phi}\right), \mathbb{E}\left(D_{\phi}\right)$ and $\operatorname{var}\left(D_{\phi}\right)$.

Similarity-based anomaly detection on test sequence $\mathcal{T}$ works as follows: if $\left|S\left(\mathcal{G}_{\phi}(\mathcal{T}), \mathcal{G}_{\phi}(\mathcal{V})\right)-\mathbb{E}\left(S_{\phi}\right)\right|>\alpha_{S}$, we raise an $S$-type alert (S-type detection); if $\left|D_{\mathrm{JS}}\left(\mathcal{Q}_{\phi}(\mathcal{T}) \| \mathcal{Q}_{\phi}(\mathcal{V})\right)-\mathbb{E}\left(D_{\phi}\right)\right|>\alpha_{D}$, we raise a $D$-type alert $(\mathbf{D}$-type detection). Using the Chebyshev's inequality, we can determine parameters $\alpha_{S}$ and $\alpha_{D}$ as follows: $\alpha_{S}=\sqrt{\operatorname{var}\left(S_{\phi}\right) / \beta_{S}}$ and $\alpha_{D}=\sqrt{\operatorname{var}\left(D_{\phi}\right) / \beta_{D}}$, where $\beta_{S}$ and $\beta_{D}$ are the upper bounds on the expected false alarm rates for S-type and D-type alerts, respectively. Both $\beta_{S}$ and $\beta_{D}$ are input parameters in practice.

From the implementation perspective, the similarity-based anomaly detection schemes do not impose high computational cost. For each SMS user, we can compute the four variables $\mathbb{E}\left(S_{\phi}\right), \operatorname{var}\left(S_{\phi}\right), \mathbb{E}\left(D_{\phi}\right)$ and $\operatorname{var}\left(D_{\phi}\right)$ based on her history trace $\mathcal{V}$ in an offline fashion. We then calculate $\alpha_{S}$ and $\alpha_{D}$ accordingly. When we perform online anomaly detection, we need to know not only $\mathbb{E}\left(S_{\phi}\right)$, $\mathbb{E}\left(D_{\phi}\right), \alpha_{S}$ and $\alpha_{D}$, but also $\mathcal{G}_{\phi}(\mathcal{V})$ and $\mathcal{Q}_{\phi}(\mathcal{V})$. Clearly, the sizes of $\mathcal{G}_{\phi}(\mathcal{V})$ and $\mathcal{Q}_{\phi}(\mathcal{V})$ depend on $\phi$. In total, the S-type detection requires at most $\phi+2$ states and the D-type detection requires at most $2 \phi+2$ states. Since $\phi$ is usually much smaller than the size of the set of unique recipients shown in history trace $\mathcal{V}$, the computational cost of our proposed scheme improves significantly compared to the aforementioned naive similarity-based scheme that demands the full knowledge of the whole history trace. Our experiments later show that $\phi=5$ is sufficient to achieve high detection accuracy. In that case, even for the D-type detection scheme, only 12 states are needed.

\section{Experimental Evaluation}

Setup. In this section, we shall evaluate the performance of our proposed detection schemes. For this, we use the same data trace discussed in Section 3 . As our detection schemes require some training data to derive a few parameters, we consider only those SMS users that have sent out at least 200 short messages. In total, there are 167 such users. Here note that the discarded user data are not relevant because of the limited SMS traffic they produce and are thus not a concern. We also use $70 \%$ of each SMS user's short messages for training and the remaining $30 \%$ for testing. Suppose that the number of training short messages is $n$. We let $h_{\min }$ be 10 and $h_{\max }$ be $\min \{30,\lfloor n / 10\rfloor\}$ in Equation (44). We believe that bounding $h$ between 10 and 30 provides a good balance between detection accuracy and latency. Further, we also bound $h$ from the upper side by 
$\lfloor n / 10\rfloor$ to ensure that there are at least 10 elements for variance computation; if we have enough training data, such a constraint can be relieved. We also have: $\beta_{R}=\beta_{H}=\beta_{S}=\beta_{D}=\beta$, and vary $\beta$ between 0.05 and 0.1 .

False positive rates. The false positive rates of the four detection schemes are shown in the following table:

\begin{tabular}{ccc} 
Scheme & $\beta=0.05$ & $\beta=0.1$ \\
\hline R-type detection & $1.0 \%$ & $2.2 \%$ \\
H-type detection & $0.8 \%$ & $2.7 \%$ \\
S-type detection & $0.0 \%$ & $5.4 \%$ \\
D-type detection & $0.0 \%$ & $4.3 \%$ \\
\hline
\end{tabular}

From the above table, we observe that the false positive rates of all four detections are very low. The effect of $\beta$ on the false positive rates is also obvious: a higher $\beta$ leads to a higher false positive rate, irrespective of the type of alerts considered. This is because a higher $\beta$ lowers threshold $\alpha_{R}$ in Equation (4) (or $\alpha_{H}, \alpha_{S}$, and $\alpha_{D}$ in the other three cases).

Detection rates of blending attacks. In our experiments, we consider every pair of SMS users in which one is the victim and the other is the attacker. Suppose that SMS user $a$ is the victim and $b$ is the attacker. We first identify the timestamp of the last message in user $a$ 's training dataset; we further get the list of messages that are sent by user $b$ that are sent after that timestamp in the trace. For brevity, we call this list an attack list. Since it is possible that the attack list may not have enough messages, e.g., because user $b$ quit from system before data collection terminated, we only consider those cases that have at least $4 h_{a}^{*}$ messages on the attack list, where $h_{a}^{*}$ is the detection window size for user $a$. Messages on the attack list are then merged with those in user $a$ 's test dataset, with their timestamp ordering unchanged as in the original trace.

Among all pairs of SMS users considered, we compute the fraction of cases in which the blending attack is successfully detected by each scheme, and the average detection delay in the number of detection windows if the attack is indeed detected. The results are as follows:

\begin{tabular}{c|cc|cc}
\hline Scheme & \multicolumn{2}{|c|}{$\beta=0.05$} & \multicolumn{2}{c}{$\beta=0.1$} \\
& Rate & Delay & Rate & Delay \\
\hline \hline R-type & $35.6 \%$ & 3.9 & $55.6 \%$ & 4.2 \\
H-type & $40.5 \%$ & 1.8 & $62.3 \%$ & 2.8 \\
S-type & $44.1 \%$ & 1.0 & $71.6 \%$ & 1.0 \\
D-type & $65.1 \%$ & 1.0 & $81.7 \%$ & 1.0 \\
\hline
\end{tabular}

Three observations can made from the above table. First, similarity-based schemes can detect blending attacks with higher rates and smaller detection delays than mean-based schemes. This is because similarity-based schemes encode more information in the detection metrics. Second, both H-type detection 
and D-type detection consider not only the set of unique recipients, but also the distribution of the number of short messages sent to each recipients. Hence, they perform better than both the R-type and S-type detection schemes. Third, a higher $\beta$ leads to a higher detection threshold, thereby improving both the detection rate and the detection delay, irrespective of the detection scheme.

Detection rates of broadcast attacks. In the experiments, we intermingle the test dataset of each SMS user with malicious messages sent to recipients that are randomly chosen from those observed in the training dataset. For each SMS user, exactly $\gamma$ malicious messages are sent out at 12:00PM every day. We call $\gamma$ the broadcast threshold, which is varied among 10, 20, 30, and 40 .

The detection ratios are depicted in Figure 13. Unsurprisingly, detection ratios when $\beta=0.1$ are higher than those when $\beta=0.05$. We note, however, that the relative ranks of the four schemes differ significantly from the detection results for blending attacks. Detection based on recipient similarity metric (i.e., S-type detection) performs the worst but detection simply based on the number of unique recipients (i.e., R-type detection) performs quite well. Recall that R-type detection for blending attacks is not as effective as the other three schemes. Such difference actually attributes to the type of attacks we are considering. For broadcasting attacks, as recipients of illegitimate short messages are actually drawn from those recipients of those legitimate short messages, the change on the recipient similarity metric under broadcasting attacks is limited. Broadcast attacks, however, generate a large number of messages with different recipients, thereby exposing themselves to the R-type detection scheme which simply monitors the change on the number of unique recipients within each detection window. On the other hand, Figure 13 reveals that D-type detection is still effective against broadcast attacks. This is because although broadcast attacks mimic the set of recipients that have been observed in the training dataset, the distribution of the number of messages sent to each recipient is still different from that in the training dataset.

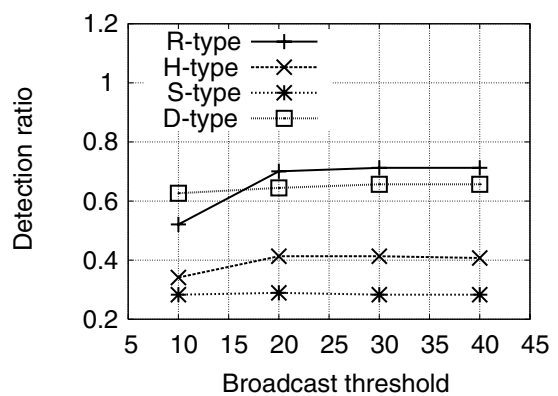

(1) $\beta=0.05$

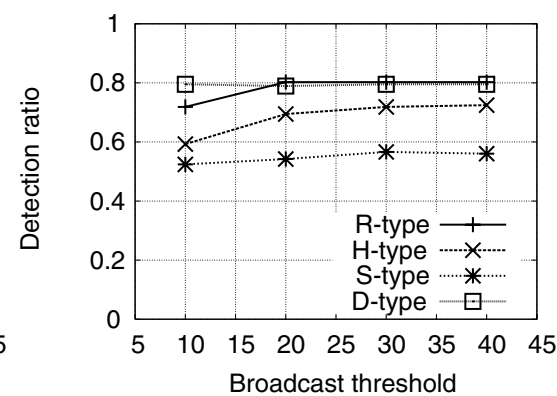

(2) $\beta=0.1$

Fig. 13. Detection ratio of broadcast attacks 
The average detection delay in the number of detection windows are given in the following table:

\begin{tabular}{|c|c|c|c|c|}
\hline$\beta$ & R-type & H-typ & 5-typ & D-type \\
\hline 0.05 & 4.0 & 3.7 & 6.8 & 4.1 \\
\hline 0.1 & 3.2 & 3.0 & 5.5 & 3.2 \\
\hline
\end{tabular}

Recall that on average similarity-based schemes detect blending attacks within a single detection window (if the detection is successful). For broadcast attacks, however, detection delays are higher because illegitimate short messages are sent at the same time in a day in our experiments and the detector thus has to wait for that moment to catch these attacks.

Hybrid detection. We now explore the detection solution space further by combining multiple detection schemes together. Due to space limitation, we consider only two hybrid detection schemes: In the first one $(\mathbf{R} / \mathbf{H} / \mathbf{S} / \mathbf{D})$, if any type of alert is flagged, we treat it as anomalous; otherwise, we treat it as normal; in the second one $(\mathbf{S} / \mathbf{D})$, if an S- or D-type of alert is flagged, we treat it as anomalous; otherwise, we treat it as normal. The following table provides the performance of these two schemes $\left(D R_{\text {blending }}\right.$ and $D R_{\text {broadcast }}$ denote the detection ratio of blending and broadcast attacks, respectively):

\begin{tabular}{c|cc|cc}
\hline & \multicolumn{2}{|c|}{$\mathbf{R} / \mathbf{H} / \mathbf{S} / \mathbf{D}$} & \multicolumn{2}{c}{$\mathbf{S} / \mathbf{D}$} \\
& $\beta=0.05$ & $\beta=0.1$ & $\beta=0.05$ & $\beta=0.1$ \\
\hline False alarm rate & $1.3 \%$ & $8.5 \%$ & $0.0 \%$ & $5.0 \%$ \\
DR $R_{\text {blending }}$ & $85.7 \%$ & $96.2 \%$ & $69.1 \%$ & $83.4 \%$ \\
$D R_{\text {broadcast }}(\gamma=10)$ & $78.3 \%$ & $94.0 \%$ & $65.7 \%$ & $82.5 \%$ \\
$D R_{\text {broadcast }}(\gamma=20)$ & $82.5 \%$ & $92.8 \%$ & $68.1 \%$ & $80.7 \%$ \\
$D R_{\text {broadcast }}(\gamma=30)$ & $83.7 \%$ & $93.4 \%$ & $69.3 \%$ & $81.9 \%$ \\
$D R_{\text {broadcast }}(\gamma=40)$ & $83.1 \%$ & $93.4 \%$ & $69.3 \%$ & $81.9 \%$ \\
\hline
\end{tabular}

We note that the $\mathbf{R} / \mathbf{H} / \mathbf{S} / \mathbf{D}$ scheme with $\beta=0.1$ can catch blending and broadcast attacks with detection ratios higher than $90 \%$ but at the expense of a relatively high false positive rate, which is about $8.5 \%$; when $\beta=0.05$, the false alarm rate is only $1.3 \%$ but its detection ratios of blending and broadcast attacks fall between $78 \%$ and $86 \%$. The $\mathbf{S} / \mathbf{D}$ scheme, although not able to detect as many attacks as the $\mathbf{R} / \mathbf{H} / \mathbf{S} / \mathbf{D}$ scheme with the same $\beta$, does not generate any false alarm when $\beta=0.05$, and still catches about two thirds of the attacks.

There is a clear tradeoff between high detection rates and low false alarm rates. In practice, the decision on which parameterized detection scheme to use can be made based on the user's preference on this tradeoff, which is also affected by the frequency at which she needs to deal with a false alarm. Here, we provide simple analysis on the average interval between two false alarms. Suppose that each detection window contains $h$ short messages and the false alarm rate is $p$. By modeling false alerts as a Bernoulli process, the average number of windows before a false alarm is raised is $1 / p$. Hence, the average number of 
messages between two false alarms is $h / p$. Consider the case with $h=20$ and $p=8 \%$. Note that we are considering a relatively high false alarm rate. Then, about every 250 short messages leads to a false alarm. In our trace, we observe that a persistent user sends 1.5 messages per day on average, suggesting that a normal SMS user needs more than 5 months on average to receive a false alarm. Even if we consider the largest average daily number of short messages sent by an SMS user in our trace, which is about 25, a false alarm is raised every 10 days.

\section{Conclusions and Future Work}

The goal of this work is to detect anomalous SMS behaviors. From an SMS trace that was collected within a five-month period, we observe that there are windowbased regularities inherent in behaviors of typical SMS users. Accordingly, we develop SMS-Watchdog, a light-weight detection scheme that relies on normal social behavior profiles built for each SMS user. Experimental results show that our detection approach can detect more than $92 \%$ of SMS-based attacks with false alarm rate $8.5 \%$, or about two thirds of the attacks without any false alarm.

Admittedly, SMS-Watchdog is not panacea for all SMS-related attacks. For instance, SMS-Watchdog is not able to detect SMS faking attacks, as such attacks simulate the behavior of SMS switches (i.e., SMSC in Figure 1) and the illegitimate SMS messages do not go through the SMSC of the originating terminal, where the SMS-Watchdog is deployed. Moreover, with the integration of the telecommunication network and the Internet, many SMS messages are now sent from the Internet. SMS accounts can be easily created through the Internet and then used to send spamming or phishing SMS messages. Given the fact that SMS-Watchdog requires a training process to build a behavioral profile for each SMS user, it is difficult for SMS-Watchdog to identify those transient SMS accounts that are used only for spamming or phishing purposes.

Moreover, as SMS-Watchdog detects abnormal SMS activities by monitoring deviations from behavioral profiles trained under normal circumstances, it is possible that some malware can intelligently evade its detection. For example, a stealthy malware can learn the behavior of an SMS user from her recent SMS communication history and then send spamming or phishing SMS messages in a similar fashion. Also, the design of SMS-Watchdog takes the variation of a typical SMS user's regular behavior into consideration to avoid high false positive rates. Accordingly, a stealthy malware can exploit this to evade its detection by limiting the number of illegitimate SMS messages sent within each detection window.

With the lesson learned from the increasing sophistication of cyber-attacks in the Internet, we do not claim that SMS-Watchdog can address all existing or future SMS-related attacks. While we will continuously improve the effectiveness of SMS-Watchdog against these attacks, we also plan to explore other complementary approaches to protect this increasingly popular service. 


\section{References}

1. Bose, A., Hu, X., Shin, K.G., Park, T.: Behavioral detection of malware on mobile handsets. In: Proceedings of MobiSys 2008 (2008)

2. Chandola, V., Banerjee, A., Kumar, V.: Anomaly detection: A survey. ACM Computing Survey (September 2009) (to appear)

3. Cover, T., Thomas, J.: Elements of Information Theory. John Wiley, Chichester (1991)

4. http://www.redherring.com/Home/19081

5. Davis, A.B., Goyal, S.K.: Knowledge-based management of cellular clone fraud. In: Proceedings of IEEE PIMRC 1992, Boston, MA, USA (1992)

6. Enck, W., Traynor, P., McDaniel, P., Porta, T.L.: Exploiting open functionality in SMS-capable cellular networks. In: Proceedings of CCS 2005 (2005)

7. Fawcett, T., Provost, F.: Activity monitoring: noticing interesting changes in behavior. In: Proceedings of ACM KDD 1999 (1999)

8. Hu, G., Venugopal, D.: A malware signature extraction and detection method applied to mobile networks. In: Proceedings of IPCCC 2007 (April 2007)

9. Kim, H., Smith, J., Shin, K.G.: Detecting energy-greedy anomalies and mobile malware variants. In: Proceedings of MobiSys (2008)

10. Lee, L.: Measures of distributional similarity. In: Proceedings of the 37th Annual Meeting of the ACL (1999)

11. Lee, W., Xiang, D.: Information-theoretic measures for anomaly detection. In: Proceedings of IEEE S\&P (2001)

12. Lin, Y., Chlamtac, I.: Wireless and Mobile Network Architectures. John Wiley \& Sons, Inc., Chichester (2001)

13. Meng, X., Zerfos, P., Samanta, V., Wong, S.H.Y., Lu, S.: Analysis of the reliability of a nationwide short message service. In: Proceedings of INFOCOM 2007 (2007)

14. Noble, C.C., Cook, D.J.: Graph-based anomaly detection. In: KDD 2003: Proceedings of the ninth ACM SIGKDD international conference on Knowledge discovery and data mining (2003)

15. http://www.vnunet.com/vnunet/news/2163586/sms-phishing-attackseen-wild

16. http://www.kval.com/news/local/17945949.html

17. http://www.mobilemarketer.com/cms/opinion/columns/1610.html

18. http://www.textually.org/textually/archives/2007/12/018482.htm

19. http://www .openmindnetworks. com/SMSSpoofing.asp

20. Stolfo, S.J., Hershkop, S., Hu, C., Li, W., Nimeskern, O., Wang, K.: Behavior-based modeling and its application to email analysis. ACM Transactions on Internet Technology 6(2), 187-221 (2006)

21. Sun, B., Yu, F., Wu, K., Xiao, Y., Leung, V.C.M.: Enhancing security using mobility-based anomaly detection in cellular mobile networks. IEEE Trans. on Vehicular Technology 55(3) (2006)

22. http://searchcio-midmarket.techtarget.com/tip/0, 289483, sid183_ gci1310706,00.html

23. Taniguchi, M., Haft, M., Hollmn, J., Tresp, V.: Fraud detection in communications networks using neural and probabilistic methods. In: Proceedings of the 1998 IEEE International Conference in Acoustics, Speech and Signal Processing (1998)

24. Traynor, P., Enck, W., McDaniel, P., Porta, T.L.: Mitigating attacks on open functionality in SMS-capable cellular networks. In: Proceedings of MobiCom 2006 (2006) 
25. Yan, G., Eidenbenz, S., Sun, B.: Mobi-watchdog: you can steal, but you can't run! In: Proceedings of ACM WiSec 2009, Zurich, Switzerland (2009)

26. Yan, G., Xiao, Z., Eidenbenz, S.: Catching instant messaging worms with changepoint detection techniques. In: LEET 2008: Proceedings of the 1st Usenix Workshop on Large-Scale Exploits and Emergent Threats, Berkeley, CA, USA (2008)

27. Zerfos, P., Meng, X., Samanta, V., Wong, S.H.Y., Lu, S.: A study of the short message service of a nationwide cellular carrier. In: Proceedings of IMC 2006 (2006) 